\title{
Charge-exchange neutral particle measurements on electron cyclotron resonance heating experiments in hot-ion-mode plasmas of the GAMMA 10 tandem mirror
}

\author{
Y. Nakashima, Y. Hasegawa, M. Shoji, ${ }^{\text {a) }}$ S. Kobayashi, T. Saito, Y. Kiwamoto, T. Cho, \\ A. Mase, M. Ichimura, A. Itakura, M. Hirata, J. Kohagura, K. Md. Islam, M. Oishi, \\ M. Yoshikawa, T. Tamano, and K. Yatsu \\ Plasma Research Center, University of Tsukuba, Tsukuba, Ibaraki 305-8577, Japan
}

(Presented on 10 June 1998)

\begin{abstract}
Charge-exchange $(\mathrm{C}-\mathrm{X})$ neutral particle measurements have been carried out in hot-ion-mode plasmas of the GAMMA 10 tandem mirror. In the present experiment, a microwave power of $40 \mathrm{~kW}$ in $28 \mathrm{GHz}$ is injected toward a second harmonic ECR layer located in the vicinity of the ICR layer at the central region and the radial profiles of ion temperatures determined from the energy spectrum of the $\mathrm{C}-\mathrm{X}$ neutrals by using a neutral particle energy analyzer (NPA) are investigated from the viewpoint of ion energy balance. At the onset of the ECRH pulse, a remarkable increase of $\mathrm{C}-\mathrm{X}$ neutral flux with high energy (few keV to few tens keV) is observed with NPA and the resultant ion temperature on the plasma axis is found to increase from 2.5 to $5.0 \mathrm{keV}$ at the electron line density of $3 \times 10^{13} \mathrm{~cm}^{-2}$. Based on the measured plasma parameters, radial profiles of ion-energy losses due to classical processes are evaluated and ECRH in the central region is confirmed to reduce the energy loss due to electron drag significantly in the core-plasma region. (C) 1999 American Institute of Physics. [S0034-6748(99)55501-9]
\end{abstract}

\section{INTRODUCTION}

Ion temperature measurement is an important subject to analyze ion energy balance and to clarify ion-heating mechanisms in magnetically confined plasmas. In the GAMMA 10 tandem mirror, ion cyclotron range of frequency (ICRF) waves have been extensively applied for sustaining/heating the plasmas and an ion temperature of $\sim 10 \mathrm{keV}$ at the electron density of $\sim 2 \times 10^{12} \mathrm{~cm}^{-3}$ has been achieved in the central cell by using a strong ion cyclotron resonance heating (ICRH) ${ }^{1}$ In the above operation regime, however, the bulk electron density is rather low (several tens eV) and collisional drag by electrons is thought to be a dominant loss channel on stored ion energy in hot-core plasmas. Recently an experiment of electron cyclotron resonance heating (ECRH) in a central cell plasma has been performed successfully, in which an effective ion heating has been confirmed. ${ }^{2,3}$ In this experiment, a microwave power of 40 $\mathrm{kW}$ in $28 \mathrm{GHz}$ is injected toward a second harmonic ECR layer located in the vicinity of the ICR layer at the central region.

A charge-exchange $(\mathrm{C}-\mathrm{X})$ neutral particle analyzer has been used for ion-temperature measurements and investigation of high-energy ions in high temperature plasmas for many years. ${ }^{4-8}$ In GAMMA $10 \mathrm{C}-\mathrm{X}$ neutral particle measurements have been carried out to analyze ion temperature of hot-ion-mode plasmas in the central cell. ${ }^{9}$ In this article we report the detailed analysis of hot ions in the ECRH experiments based on $\mathrm{C}-\mathrm{X}$ neutral particle measurements and ion-energy balance analyses. A series of the ECRH ex-

${ }^{a)}$ Also at: National Institute for Fusion Science, Toki 509-5292, Japan. periments provides a comparison with the measured results in only ICRF-heated plasmas at the same extent of stored ion energy in order to investigate the ECRH effect. The radial profile of ion-energy loss in each experiment is also evaluated based on the measured results.

\section{EXPERIMENTAL APPARATUS}

GAMMA 10 is a minimum-B anchored tandem mirror with thermal barrier produced at a plug/barrier region in each mirror end, which consists of an axisymmetric central mirror cell, anchor cells with ying-yang coils for MHD stabilization, and plug/barrier cells with axisymmetric mirror cells for producing end-plugging potentials. ${ }^{10,11}$ The length of the central cell is $6 \mathrm{~m}$ and the magnetic strength is normally 0.43 $\mathrm{T}$ and is varied from 0.3 to $0.57 \mathrm{~T}$. Initial plasma is injected from both ends by plasma guns and is built up with two types of ICRF waves together with gas puffing. One of the ICRF waves (RF1) is mainly used for MHD stabilization and excited by so-called NAGOYA type III antenna. Another wave (RF2) is used for heating the central-cell ions by ion cyclotron damping near the midplane of the central cell, which is excited by a pair of the double half-turn antennas.

A six-channel neutral particle energy analyzer (NPA) is installed perpendicularly to the magnetic field line near the midplane of the central cell. The structural view of the analyzer and the vertical view of the NPA system are shown in Fig. 1. NPA is a parallel-plate-type electrostatic energy analyzer with a gas stripping cell which analyzes neutral particles from the energy of 1 to $30 \mathrm{keV}$. Hydrogen gas is used for charge stripping of neutrals. As shown in Fig. 1(b) NPA also covers the whole cross section of the plasma radius by scanning the measuring angle vertically shot by shot $\left(\theta_{\mathrm{NPA}}\right.$ 
(a)

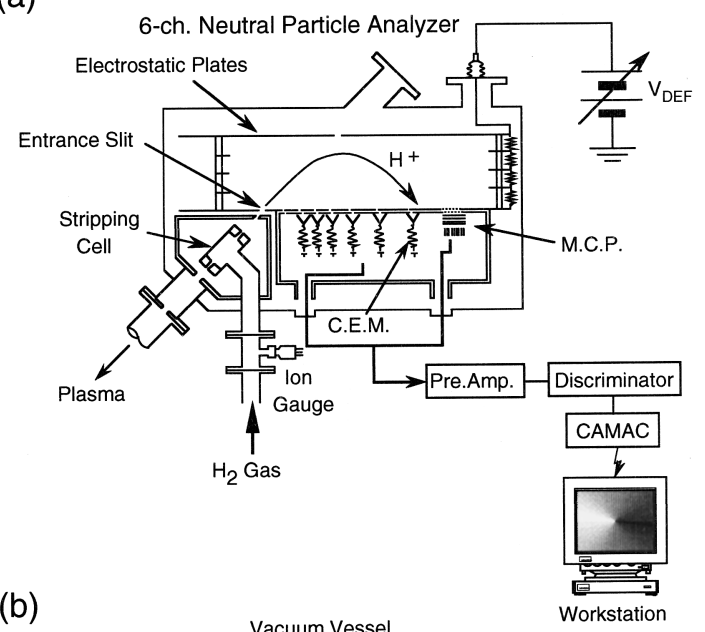

(b)

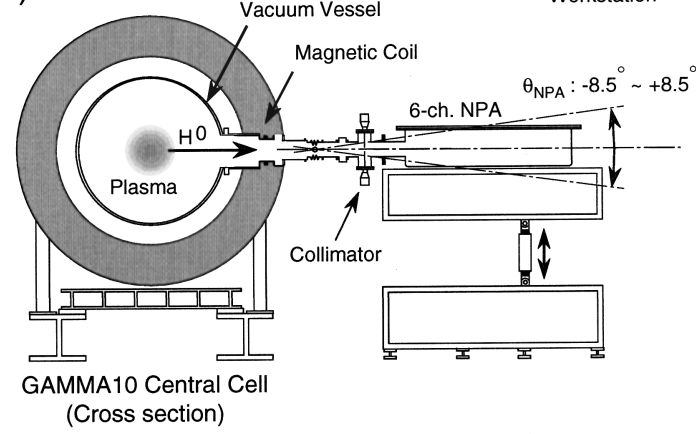

FIG. 1. The structural view of six-channel neutral particle analyzer (NPA) and the vertical view of the NPA system.

$=+8.5^{\circ}$ to $-8.5^{\circ}$, which provides radial $\mathrm{C}-\mathrm{X}$-flux profiles of perpendicularly heated ions. The viewing area of NPA is 2 $\mathrm{cm}$ in the vertical direction and $5 \mathrm{~cm}$ in the horizontal direction at the plasma center. Secondary ions analyzed through the electrostatic plates are detected with channeltron-type electron multipliers (MURATA Co. EMS6089B) in pulsecounting mode. Detected pulses of $\mathrm{C}-\mathrm{X}$ neutrals are accumulated in every $0.5 \mathrm{~ms}$ and transferred to a CAMAC system and finally analyzed with a workstation. An absolute calibration of the analyzer has been carried out by using an atomic hydrogen beam and the basic characteristics in each analyzer channel, such as the relationship between the particle energy and the deflection voltage applied to the electrostatic plates, the energy resolution, etc., have been measured in the energy range from 1 to $30 \mathrm{keV}^{12}$

\section{EXPERIMENTAL RESULTS}

Figure 2 shows the temporal behavior of plasma parameters obtained in ECRH experiments. On the left-hand side of the figure, the results measured in the case of applying the central ECRH (C-ECRH) from $t=115$ to $145 \mathrm{~ms}$ are represented and it is observed that the diamagnetic signal and a soft x-ray signal measured at the central cell increase remarkably with the C-ECRH pulse. For comparison with the above results an experiment using a subpulse in the RF2 oscillator power (RF2-sp) is carried out in which the ICRF power is raised stepwise so as to achieve the same extent of the diamagnetic signal in the C-ECRH experiment as shown
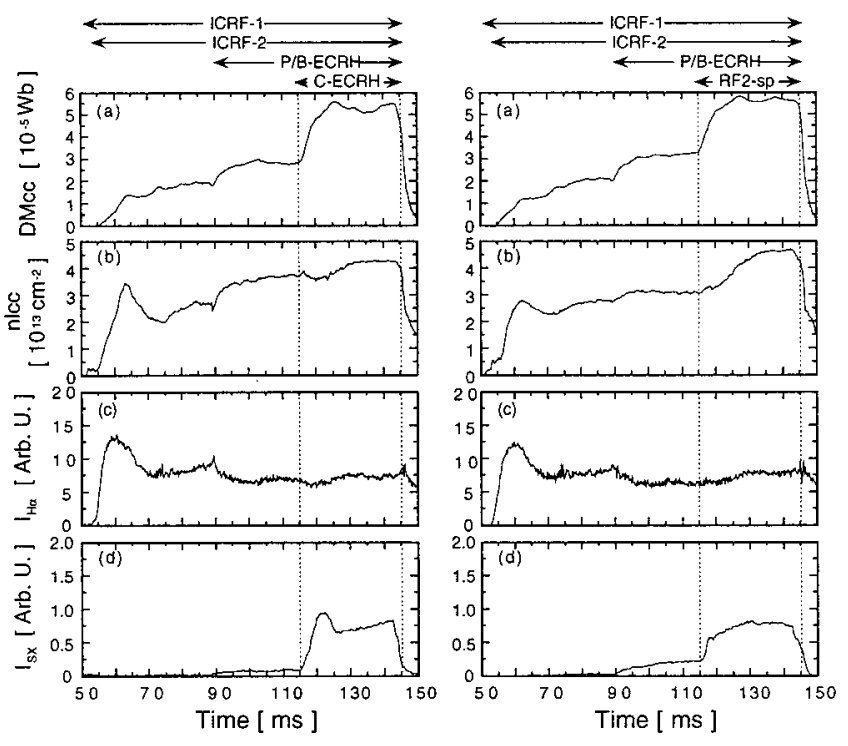

FIG. 2. Temporal behavior of typical plasma parameters in the central ECRH experiment (left-hand side) and the ICRF2 subpulse experiment (right-hand side). The diamagnetic signal (a), electron line density (b), intensity of the $\mathrm{H} \alpha$ line emission (c), and the soft x-ray signal (d).

in the right-hand side of Fig. 2. Although an increase of the electron line density is observed by $30 \%$, a temperature increase corresponding to RF2-sp is clearly confirmed in comparison with the increase of diamagnetic signal.

Time evolution of the $\mathrm{C}-\mathrm{X}$ neutral flux in the energy range of 3.9-19.9 keV measured with NPA is shown in Fig. 3 . The $\mathrm{C}-\mathrm{X}$ neutral flux in the higher energy range increases rapidly at the onset of heating pulses in both experiments (C-ECRH and RF2-sp). Note that a significant delay in the $\mathrm{C}-\mathrm{X}$ neutral flux with the energy of $19.9 \mathrm{keV}$ is observed in the C-ECRH experiment, while the flux in the RF2-sp experiment begins to increase immediately after the onset of the subpulse. The energy spectra of hot ions in the central cell obtained from the $\mathrm{C}-\mathrm{X}$ neutral flux before $(t$ $=100 \mathrm{~ms})$ and during $(t=130 \mathrm{~ms})$ the heating pulses (CECRH and RF2 $-s p$ ) are represented in Figs. 3(d) and 3(e). In both cases significant changes in the energy spectrum are observed, which indicates the increase of ion temperature due to the heating pulses.

Figure 4 shows the radial profiles of plasma parameters measured in quasi-steady state phases before and during heating pulses. The upper figures represent the ion and electron temperatures which are obtained from the $\mathrm{C}-\mathrm{X}$ neutral particle and soft $\mathrm{x}$-ray measurements, respectively. For the determination of the radial profile of the ion temperature a numerical calculation is also used in which the effect of the finite Larmor motion of hot ions and molecular hydrogen in the plasma are taken into consideration and its uncertainty has been evaluated to be within $10 \%$ at the core region. ${ }^{13}$ The radial profiles of the electron density are shown in the bottom figures together with atomic and molecular hydrogen densities determined from $\mathrm{H} \alpha$ measurements and neutral transport simulation by using a modified DEGAS code. ${ }^{14,15}$ The quantity of diamagnetism calculated from these temperature and density profiles is confirmed to agree with the 

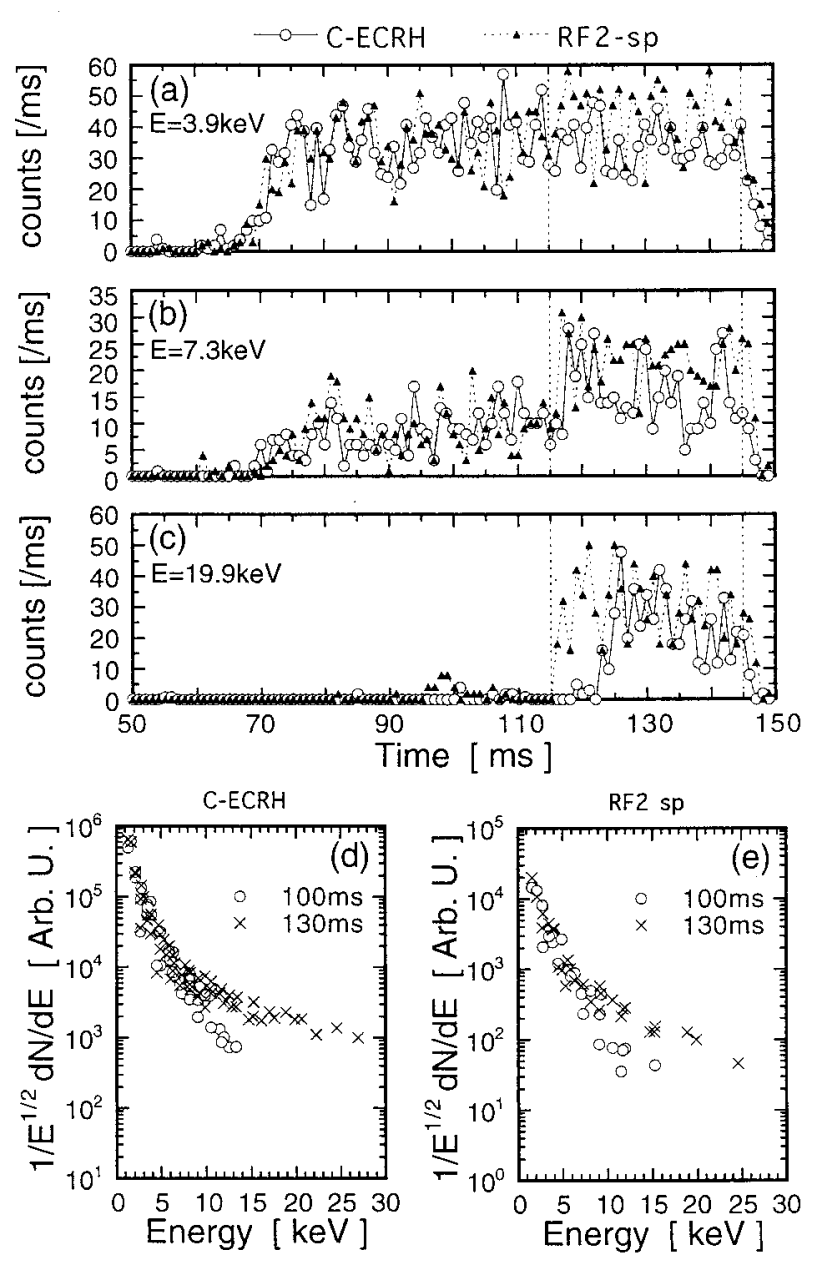

FIG. 3. Time evolution of charge-exchange neutral flux with energy $3.9 \mathrm{keV}$ (a), $7.3 \mathrm{keV}$ (b), and $19.9 \mathrm{keV}$ (c). Energy spectra of hot ions in the CECRH shot (d) and the RF2-sp shot (e).

result obtained from the diamagnetic loop in the central cell, which also supports the reliability of the above analysis. As shown in the figure ion temperatures on axis are nearly doubled in both cases by each heating pulse $(2.5-5.0 \mathrm{keV}$ in C-ECRH, 2.6-4.6 keV in RF2-sp) and the profiles are observed to become steep. While the electron temperature is heated more remarkably in the C-ECRH experiment (55-140 $\mathrm{eV})$ than in the RF2 $-s p$ experiment $(55-80 \mathrm{eV})$, since CECRH is a direct electron-heating method.

\section{EVALUATION OF ION-ENERGY LOSSES}

Based on the measured results of plasma parameters the radial profiles of various ion-energy losses are evaluated. In Fig. 5 radial profiles of collisional loss due to electrons (electron drag) $P_{e i}, \mathrm{C}-\mathrm{X}$ loss with hydrogen atoms, $P_{\mathrm{cxH}}$, and $\mathrm{C}-\mathrm{X}$ loss with hydrogen molecules, $P_{\mathrm{cxH} 2}$, are represented under two quasi-steady state conditions and compared between the two plasma heating methods. Before applying the heating pulses shown in the upper figures $(t=100 \mathrm{~ms})$, the ion energy loss to electrons is dominant in the hot-core region, since the electron temperature is much lower than ion temperature. It is found that such energy loss due to electrons is reduced by about $9 \%$ with C-ECRH $(t=130 \mathrm{~ms})$ as shown

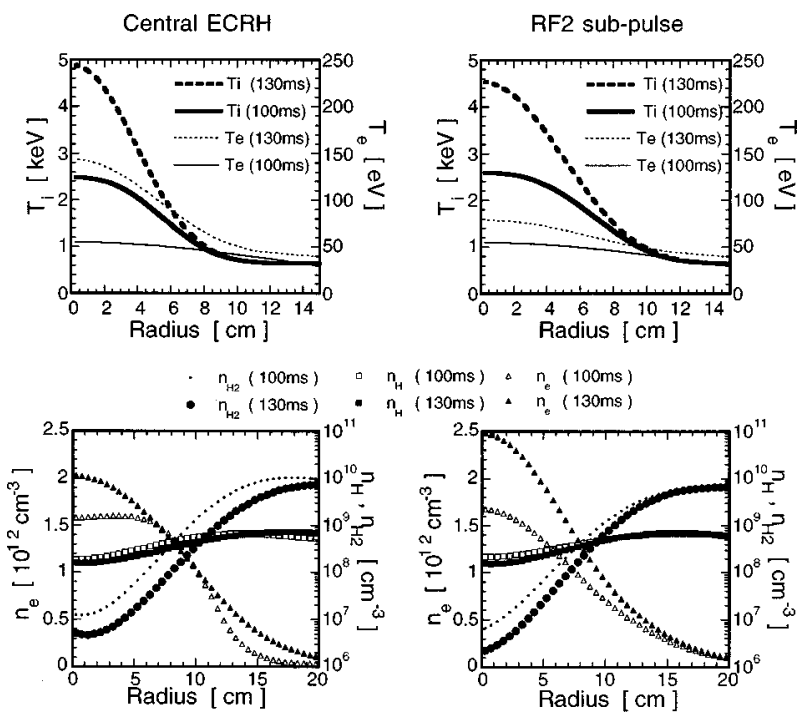

FIG. 4. Radial profiles of plasma parameters in the central ECRH experiment (left-hand side) and the ICRF2 subpulse experiment (right-hand side).

in the bottom figure of Fig. 5(a). The increase of C-X loss due to the ion temperature increase is also recognized and the total amount of ion-energy loss is estimated to increase by $\sim 20 \%$ on the plasma axis. In the case of RF2-sp experiments, on the other hand, both losses due to electron drag and charge exchange are nearly doubled and the total-loss amount is also doubled. This result is ascribed to the lesser increase of the electron temperature in the RF2-sp experiments than in C-ECRH, since the electron drag power is mainly affected by electron temperature $\left(\propto T_{e}^{-3 / 2}\right)$.

Using the radial profiles of $P_{\text {loss }}$ shown in Fig. 5, the integrated energy loss within the hot-core region is calculated. In this calculation an area in which hot ions are contained is assumed to be $\leqslant 300 \mathrm{~cm}$ in axial length and $\leqslant 10 \mathrm{~cm}$ in radial direction considering the axial distribution of the diamagnetic signal. The C-ECRH pulse reduces the colli- (a) $\mathrm{C}$-ECRH

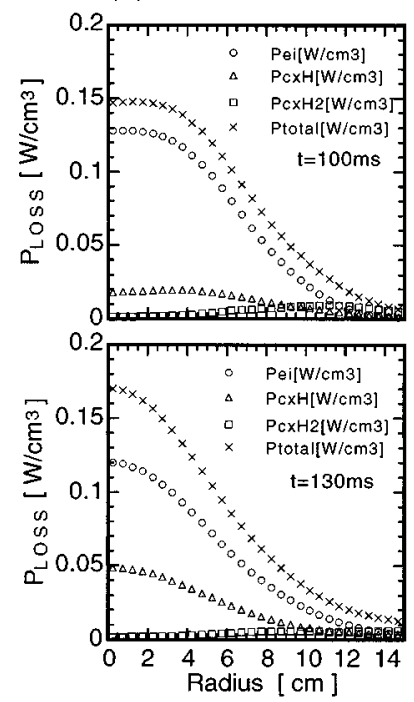

(b) RF2-sp

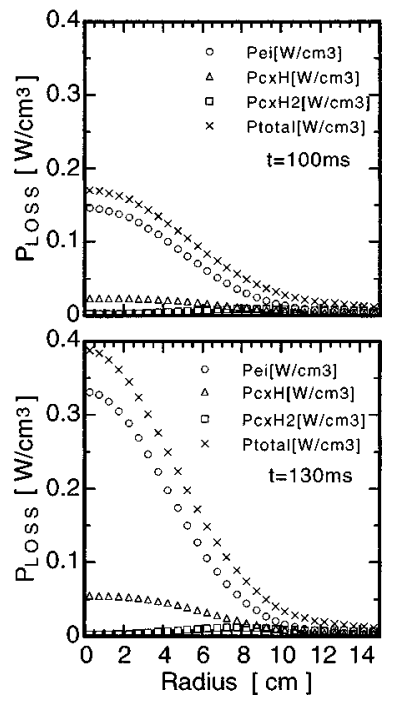

FIG. 5. Radial profile of ion-energy loss in the central ECRH experiment (a) and the ICRF2 subpulse experiment (b). Note that the vertical scale in (b) is doubled. 
sional loss due to electrons by more than $20 \%$ and the total energy loss decreases by $10 \%$. In the case of RF2-sp, on the other hand, the collisional loss to electron increases by $84 \%$, which leads to the increase of $80 \%$ in total ion-energy loss. The above results show that C-ECRH increases the electron temperature and reduces electron-drag loss that is a dominant loss channel in the central cell plasma of GAMMA 10 and that such a direct electron-heating method finally leads to the effective ion heating.

The diamagnetic signal intensity increases by factor 1.8 in C-ECRH experiments as shown in Fig. 2. The amount of this increase should contain a contribution from electrons. The contribution from the bulk electron is negligibly small compared with hot ions, while hot electrons may be significant. The ratio of the ion component to the total diamagnetism is calculated to be $80 \%$ by using the measured ion temperature. The contribution of hot electron component is estimated to be $15 \%$ of total diamagnetism based on the $\mathrm{x}$-ray measurements. Consequently the increase of the diamagnetism observed in C-ECRH is explained quantitatively within the error of the measurements.

\section{CONCLUSIONS}

Charge-exchange neural particles have been measured in the central ECRH experiment of the hot-ion-mode plasmas. A significant increase of the $\mathrm{C}-\mathrm{X}$ neural flux was observed during the $\mathrm{C}$-ECRH pulse and the radial profile of ion temperature was determined. Ion temperature was estimated to be doubled, which clearly showed that the direct bulkelectron heating using second harmonic ECR was able to lead to effective ion heating. Detailed calculation of the radial profile of ion-energy loss provided a $10 \%$ reduction of ion-energy loss due to the C-ECRH pulse. An evaluation of the increase in the diamagnetic signal due to C-ECRH showed that $80 \%$ of total diamagnetism contributed to the increase of ion energy within the experimental errors.

\section{ACKNOWLEDGMENTS}

The authors would like to acknowledge the members of the GAMMA 10 group, University of Tsukuba, for their collaboration in the experiments.

${ }^{1}$ T. Tamano, Phys. Plasmas 2, 2321 (1995).

${ }^{2}$ T. Saito et al., Proceedings 16th International Conference on Fusion Energy, Montreal, 1996 (IAEA, Vienna, 1997), Vol. 2, p. 105.

${ }^{3}$ Y. Kiwamoto et al., Plasma Phys. Controlled Fusion 39, 381 (1997).

${ }^{4}$ V. V. Afrosimov and I. P. Glakkovskii, Sov. Phys. Tech. Phys. 12, 1135 (1967).

${ }^{5}$ H. Takeuchi, A. Funahashi, K. Takahashi, H. Shirakata, and S. Yano, Jpn. J. Appl. Phys. 16, 139 (1977).

${ }^{6}$ H. Zushi, Y. Nakashima, K. Kondo, A. Iiyoshi, and K. Uo, J. Phys. Soc. Jpn. 51, 2673 (1982).

${ }^{7}$ S. L. Davis, D. Mueller, and C. J. Keane, Rev. Sci. Instrum. 54, 315 (1983).

${ }^{8}$ A. L. Roquemore, G. Gammel, G. W. Hammett, R. Kaita, and S. S. Medley, Rev. Sci. Instrum. 56, 1120 (1985).

${ }^{9}$ Y. Nakashima et al., Fusion Eng. Des. 34-35, 555 (1997).

${ }^{10}$ M. Inutake et al., Phys. Rev. Lett. 55, 939 (1985).

${ }^{11}$ T. Tamano et al., Proceedings 15th International Conference on Plasma Physics and Controlled Nuclear Fusion Research, Seville, 1994, (IAEA, Vienna, 1995), Vol. 2; p. 399.

${ }^{12}$ M. Shoji, Ph.D. thesis, University of Tsukuba, 1995.

${ }^{13}$ M. Shoji, Y. Nakashima, K. Yatsu, K. Tsuchiya, Y. Fukuchi, M. Ichimura, M. Inutake, and T. Tamano, J. Phys. Soc. Jpn. 65, 2846 (1996).

${ }^{14}$ D. Heifetz, D. Post, M. Petravic, J. Weisheit, and G. Bateman, J. Comput. Phys. 46, 309 (1982).

${ }^{15}$ Y. Nakashima et al., J. Nucl. Mater. 196-198, 493 (1992); Y. Nakashima et al., ibid. 241-243, 1011 (1997). 